\title{
Six-Degrees-of-Freedom Remote Actuation of Magnetic Microrobots
}

\author{
Eric Diller*, Joshua Giltinan*, Guo Zhan Lum*, Zhou Ye, and Metin Sitti
}

\begin{abstract}
Existing remotely-actuated microrobots powered by magnetic coils far from the workspace exhibit a maximum of only five-degrees-of-freedom (DOF) actuation, as creation of a driving torque about the magnetization axis is not achievable. This lack of orientation control limits the effectiveness of existing microrobots for precision tasks of object manipulation and orientation for advanced medical, biological and micro-manufacturing applications. This paper presents a magnetic actuation method that allows these robots to achieve full six-DOF actuation by allowing for a non-uniform magnetization profile within the microrobot body. This non-uniform magnetization results in additional rigid-body torques to be induced from magnetic forces via a moment arm. A general analytical model presents the working principle for continuous and discrete magnetization profiles. Using this model, microrobot design guidelines are introduced which guarantee six-DOF actuation capability. Several discrete magnetization designs which possess reduced coupling between magnetic forces and induced rigid-body torques are also presented. A simple permanent-magnet decoupled prototype is fabricated and used to quantitatively demonstrate the accuracy of the analytical model in a constrained-DOF environment and qualitatively for free motion in a viscous liquid three-dimensional environment. Results show that desired forces and torques can be created with high precision and limited parasitic actuation, allowing for full sixDOF actuation using limited feedback control.
\end{abstract}

\section{INTRODUCTION}

Remote magnetic actuation provides advantages over other mobile microrobot actuation methods in its ability to apply relatively large forces and torques at a distance. It can penetrate through most materials, including biological materials for potential applications in microfluidics [3, 9, 24], microfactories $[5,10]$, bioengineering $[11,18,20]$, and healthcare $[1,4,6$, $12,13,14,15,19,21,22,23]$. However, magnetic actuation of permanent or non-permanent (soft) magnets operating far from the driving coils have been limited to only five-degreesof-freedom (DOF) actuation, while other actuation methods often have even fewer available DOF. In particular, previous magnetic actuation schemes have not been able to apply magnetic torque about the magnet's magnetization axis. This limitation prevents a robotic element from achieving every possible orientation in its workspace. As microrobots and their applications become more complex, this limitation will increasingly hinder their motion and manipulation dexterity.

\footnotetext{
* Equally contributing authors

This work is supported by the National Science Foundation under NSF-NRI Award Number 1317477.

E. Diller, J. Giltinan, G. Z. Lum, Z. Ye, and M. Sitti are with the Department of Mechanical Engineering, Carnegie Mellon University, Pittsburgh, PA 15238, USA. E. Diller is currently with the Department of Mechanical and Industrial Engineering, University of Toronto, Toronto, ON, Canada. G. Z. Lum is also with the School of Mechanical and Aerospace Engineering, Nanyang Technological University, Singapore. M. Sitti is also currently with the MaxPlanck Institute for Intelligent Systems, 70569 Stuttgart, Germany.

Corresponding author: M. Sitti, sitti@cmu.edu, sitti@is.mpg.de.
}

Due to the limitation on orientation control, current magnetic microrobots have often been designed to be symmetrical about their magnetization axis. Such axisymmetric designs can eliminate the need for rotation control about a single axis. For example, magnetic microrobots have punctured blood vessels with a cylindrical syringe [12] and non-contact manipulators have used spherical symmetry to create predicted stokes flows [24]. However, as microrobots become available for more advanced applications, the demands on their functionality have been increasing. In order to match these expectations, the microrobots will be required to achieve six-DOF dexterity similar to that demonstrated by large-scale robotic manipulators. Centimeter-scale magnetic devices have achieved sixDOF motion by actuating multiple discrete magnets with nonuniform magnetic fields [17]. However, the underlying theory of magnetic microrobots often assumes the magnetic field is uniform in the microrobot workspace. Thus, this principle cannot be applied to microrobots in a uniform workspace.

We present here a magnetic actuation method which can overcome the challenges seen by previous magnetic actuation schemes to enable six-DOF magnetic actuation. Previous studies in magnetic microrobot actuation approximated the microrobot as a point dipole. The dipole will align to an external magnetic field, but there is no mechanism to generate a torque about the dipole axis. Here, we relax this condition. By allowing for varying magnetization in the volume of the magnet, additional coupling is encountered between applied magnetic forces and their induced rigid-body torques. This allows for the creation of rigid-body torques about any axis, without loss of actuation force command.

In this paper, we first formulate the six-DOF actuation concept for a general magnetic body with distributed arbitrary magnetization profile, and the design requirement to achieve the desired six-DOF actuation. This is followed by discussion of the case of multiple discrete magnetic elements, each with uniform magnetization. For the discrete magnet case, we show that several magnet arrangements are possible which reduce the coupling between magnetic forces and torques and their corresponding rigid-body forces and torques. This allows for a simpler mathematical description of the actuation. Finally, we experimentally validate the analytical model through reducedDOF water surface experiments and demonstrate full six-DOF motion in a liquid environment.

\section{CONCEPT}

\section{A. Magnetic Force and Torque}

Magnetic forces and torques are assumed to be applied to actuate a microrobot using magnetic fields applied from electromagnet coils outside the workspace. It is also assumed 


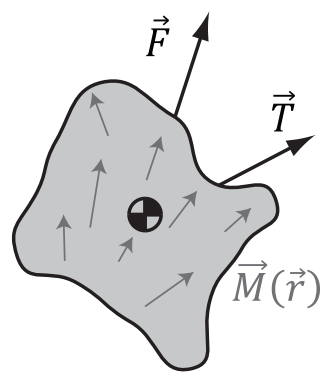

Fig. 1. Schematic of a general magnetic microrobot with distributed magnetization profile, showing the magnetic force $\vec{F}$ and total torque $\vec{T}$ exerted on the rigid body about the center of mass.

that the fields and their spatial gradients in the workspace can be generated independently while obeying Gauss's and Ampere's laws. A general magnetic microrobot with spatiallydistributed non-uniform magnetization profile $\vec{M}=\vec{M}(\vec{r})$ is shown in Fig. 1. Here $\vec{r}$ denotes the position of a point within the magnet relative to the center of mass (COM). The net equivalent magnetic moment, $\vec{m}_{e}$, can be defined as $\vec{m}_{e}=\int \vec{M} d V$. The magnetic force distribution $\vec{f}(\vec{r})$ exerted on a microrobot over the volume $V$ in a magnetic field $\vec{B}=\left[\begin{array}{lll}B_{x} & B_{y} & B_{z}\end{array}\right]^{\mathrm{T}}$, assuming no electric current is flowing in the workspace, and the generated fields and their spatial gradients are uniform, is integrated to give the total magnetic force $\vec{F}$, and is given by

$$
\begin{aligned}
& \vec{F}=\int \vec{f}(\vec{r}) d V \\
& =\int(\vec{M} \cdot \nabla) \vec{B} d V \\
& =\int\left(\begin{array}{lll}
\frac{\partial \vec{B}}{\partial x} & \frac{\partial \vec{B}}{\partial y} & \frac{\partial \vec{B}}{\partial z}
\end{array}\right)^{\mathrm{T}} \vec{M} d V .
\end{aligned}
$$

Similarly, the magnetic torque $\vec{T}_{m}$ is given by

$$
\vec{T}_{m}=\int \operatorname{sk}(\vec{M}) \vec{B} d V=\operatorname{sk}\left(\vec{m}_{e}\right) \vec{B},
$$

where $\operatorname{sk}(\vec{M})$ and $\operatorname{sk}\left(\vec{m}_{e}\right)$ are the skew symmetric matrix forms of $\vec{M}$ and $\vec{m}_{e}$, respectively. As $\vec{T}_{m}$ is generated via a cross product, it is impossible to create a torque that is parallel to $\vec{m}_{e}$. Thus existing magnetic microrobots cannot achieve full six-DOF motion.

A microrobot with non-uniform magnetization will experience unbalanced magnetic forces which will induce a rigid-body torque. The magnetic torque due to the off-axis magnetization is

$$
\begin{aligned}
\vec{T}_{o} & =\int \operatorname{sk}(\vec{r}) \vec{f}(\vec{r}) d V \\
& =\int \operatorname{sk}(\vec{r})\left(\begin{array}{lll}
\frac{\partial \vec{B}}{\partial x} & \frac{\partial \vec{B}}{\partial y} & \frac{\partial \vec{B}}{\partial z}
\end{array}\right)^{\mathrm{T}} \vec{M} d V
\end{aligned}
$$

In this study, we are particularly interested in the creation of torque about the $\vec{m}_{e}$ axis (the missing sixth-DOF). The magnitude of the torque about $\vec{m}_{e}$ generated by $\vec{T}_{o}, T_{6-\mathrm{DOF}}$, can be expressed as

$$
T_{6-\mathrm{DOF}}=\vec{T}_{o} \cdot \hat{\vec{m}}_{e}=\vec{E}^{\mathrm{T}} \vec{B}_{\mathrm{grad}},
$$

where $\vec{E}^{\mathrm{T}}=\left[\begin{array}{lllll}e_{1} & e_{2} & e_{3} & e_{4} & e_{5}\end{array}\right]$ are coefficients that are functions of $\vec{M}$ and $\vec{r}$. $\vec{B}_{\text {grad }}$ are the independent spatial gradients of $\vec{B}$, and $\hat{\vec{m}}_{e}$ is a unit vector parallel to $\vec{m}_{e}$. Without any loss of generality, there exists a local frame such that the vector $\vec{m}_{e}$ can be expressed as $\left[\begin{array}{lll}0 & 0 & m_{e}\end{array}\right]^{\mathrm{T}}$ and the spatial gradients in the local frame are given as $\vec{B}_{\text {grad }}=\left[\frac{\partial B_{x}}{\partial z} \frac{\partial B_{y}}{\partial z} \frac{\partial B_{z}}{\partial z} \frac{\partial B_{y}}{\partial y} \frac{\partial B_{x}}{\partial y}\right]^{\mathrm{T}}$. The local frame is intuitive for the design of a robot independent of the actuation coordinates. The generated torques and forces on the magnet, $\vec{T}_{m}, \vec{F}$ and $T_{6-\mathrm{DOF}}$ can be expressed in this local frame as

$$
\begin{aligned}
{\left[\begin{array}{c}
\vec{T}_{m} \\
\vec{F} \\
T_{6-\mathrm{DOF}}
\end{array}\right]_{\mathrm{L}} } & =\left[\begin{array}{ccc|ccccc}
0 & -m_{e} & 0 & 0 & 0 & 0 & 0 & 0 \\
m_{e} & 0 & 0 & 0 & 0 & 0 & 0 & 0 \\
0 & 0 & 0 & 0 & 0 & 0 & 0 & 0 \\
\hline 0 & 0 & 0 & m_{e} & 0 & 0 & 0 & 0 \\
0 & 0 & 0 & 0 & m_{e} & 0 & 0 & 0 \\
0 & 0 & 0 & 0 & 0 & m_{e} & 0 & 0 \\
\hline 0 & 0 & 0 & e_{1} & e_{2} & e_{3} & e_{4} & e_{5}
\end{array}\right]\left[\begin{array}{c}
\vec{B} \\
\vec{B}_{\text {grad }}
\end{array}\right]_{\mathrm{L}} \\
& =\mathbf{D}\left[\begin{array}{c}
\vec{B} \\
\vec{B}_{\text {grad }}
\end{array}\right]_{\mathrm{L}}
\end{aligned}
$$

Here, $\left[\begin{array}{ll}\vec{B}^{\mathrm{T}} & \vec{B}_{\text {grad }}^{\mathrm{T}}\end{array}\right]_{\mathrm{L}}^{\mathrm{T}}$ are also expressed in this local frame (denoted by the subscript L) and the matrix $\mathbf{D}$ is defined as the design requirement matrix. Note that the elements in the third row of $\mathbf{D}$ are all zero, as it indicates the limitation of existing five-DOF robots, i.e. they cannot exert any torque about the magnetization axis. Therefore, in order to overcome this limitation and achieve six-DOF actuation capabilities, the last row of $\mathbf{D}$ must be linearly independent of the other rows such that $\operatorname{rank}(\mathbf{D})$ will be six. If this rank condition is satisfied, it will then be possible to generate a non-zero $T_{6 \text {-DOF }}$ to the robot without losing any other DOF. This condition implies that either the coefficients $e_{4}$ or $e_{5}$ in the last row of $\mathbf{D}$ must be non-zero. In a physical sense, this dictates that it is necessary for the microrobot's magnetization profile to contain components which are non-parallel to $\vec{m}_{e}$. When $\operatorname{rank}(\mathbf{D})$ is six, it is possible for the total torque acting on the microrobot, $\vec{T}$, to be about any axis without actuation coupling between the applied force and the desired torque. The torque can be expressed as

$$
\vec{T}=\vec{T}_{m}+\vec{T}_{o}
$$

While the components of $\vec{T}_{o}$ orthogonal to $\vec{m}_{e}$ will contribute to the overall torque, a solution to $\vec{T}_{m}$ can be found to compensate these terms for a desired $\vec{T}$.

\section{B. Control of Magnetic Fields and Gradients}

The magnetic field and its spatial gradients depend linearly on the currents through the coils [12], and so the field and gradient terms can be expressed as

$$
\begin{gathered}
\vec{B}=\mathbf{B} \vec{I}, \\
\frac{\partial \vec{B}}{\partial x}=\mathbf{B}_{x} \vec{I} ; \quad \frac{\partial \vec{B}}{\partial y}=\mathbf{B}_{y} \vec{I} ; \quad \frac{\partial \vec{B}}{\partial z}=\mathbf{B}_{z} \vec{I},
\end{gathered}
$$


where each element of $\vec{I}$ is current through each of the $c$ coils, $\mathbf{B}$ is a $3 \times c$ matrix mapping these coil currents to the magnetic field vector $\vec{B}$ and $\mathbf{B}_{x}, \mathbf{B}_{y}, \mathbf{B}_{z}$ are the $3 \times c$ matrices mapping the coil currents to the magnetic field spatial gradients in the $x-, y$-, and $z$-directions, respectively. These mapping matrices are calculated for a given coil arrangement by treating the coils as magnetic dipoles in space and are calibrated through workspace measurements $[12,16]$. We can combine eqs. (1) and (6) to arrive at

$$
\begin{aligned}
{\left[\begin{array}{l}
\vec{T} \\
\vec{F}
\end{array}\right] } & =\left[\begin{array}{c}
\vec{M}^{\mathrm{T}} \mathbf{B}_{x} \\
\left.\operatorname{sk}\left(\vec{m}_{e}\right) \mathbf{B}+\int \operatorname{sk}(\vec{r})\left[\begin{array}{c}
\vec{M}^{\mathrm{T}} \mathbf{B}_{y} \\
\vec{M}^{\mathrm{T}} \mathbf{B}_{z}
\end{array}\right] d V\right] \vec{I} \\
\vec{m}_{e}^{\mathrm{T}} \mathbf{B}_{x} \\
\vec{m}_{e}^{\mathrm{T}} \mathbf{B}_{y} \\
\vec{m}_{e}^{\mathrm{T}} \mathbf{B}_{z}
\end{array}\right] \\
& =\mathbf{A} \vec{I},
\end{aligned}
$$

where $\mathbf{A}$ is the $6 \times c$ matrix mapping the coil currents $\vec{I}$ to the torque $\vec{T}$ and force $\vec{F}$. If the magnetization profile of the robot ensures that the $\operatorname{rank}(\mathbf{D})$ in eq. (5) is six and the number of coils $c$ is greater than or equal to 6, eq. (9) can be solved because A will also be full rank. The full solution can be accomplished for $c \neq 6$ through the pseudo-inverse, which finds the solution that minimizes the 2-norm of $\vec{I}$ as

$$
\vec{I}=\mathbf{A}^{+}\left[\begin{array}{l}
\vec{T} \\
\vec{F}
\end{array}\right] \text {. }
$$

If $c<6$, then the solution will be a least-squares approximation. Having greater than 6 coils leads to a better conditioned $\mathbf{A}$ matrix, which means a more isotropic workspace, reduction of singularity configurations and lower coil current requirements.

With knowledge of the orientation state of the microrobot, one can then solve eq. (9) in order to achieve any desired actuation force and torque.

\section{Discrete Magnet Configurations}

In order to reduce the fabrication complexity, the distributed magnetization profile can be simplified into discrete elements with uniform magnetization. To simplify the model, we assume that such discrete magnet elements can be modeled as magnetic dipoles. Three different discrete models (two-, three-, and seven-magnet configurations) will be introduced and discussed in this section. Every configuration will consist of one main magnet that permits the microrobot to achieve the usual five-DOF, and additional auxiliary magnets which enable the sixth-DOF. The microrobot orientation is defined by the orientation of the main magnet $\vec{m}$.

Similar to the continuous magnetization profile case discussed in the previous section, any combination of discrete magnets which satisfy the requirement of $\operatorname{rank}(\mathbf{D})=6$ in eq. (5) will result in six-DOF actuation capability. To get the most actuation authority, discrete magnets should be placed perpendicular to each other so as to maximize the torque about $\vec{m}$. We introduce this as the two-magnet configuration. (a) two-magnets

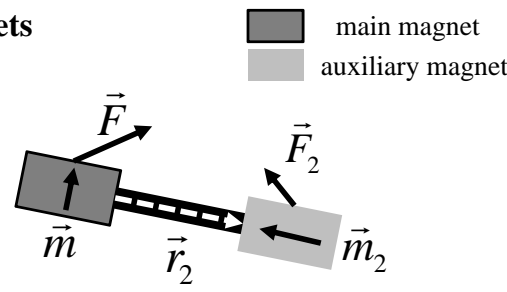

(b) three-magnets

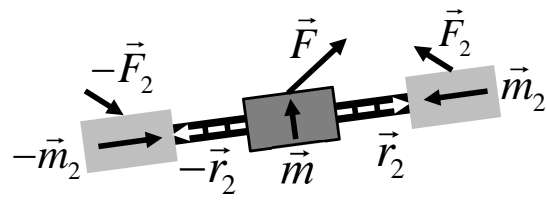

(c) seven-magnets

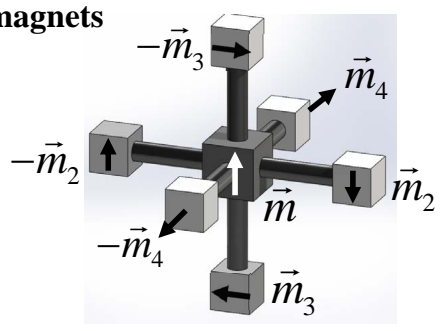

Fig. 2. The schematic of the two-, three-, and seven-magnet configurations are shown in (a), (b) and (c) respectively. In all configurations, the main magnet's magnetization vector is represented by $\vec{m}$ while the rest of the magnets are auxiliary magnets. In (b), the vectors $\vec{m}$ and $\vec{m}_{2}$ are orthogonal to one another and the magnets are placed collinearly. The auxiliary magnets have equal and opposite magnetization vectors.

\section{A. Two-magnet Configuration}

The simplest configuration, the two-magnet configuration, is illustrated in Fig. 2(a) where two magnets of magnetic dipole strength $\vec{m}$ and $\vec{m}_{2}$ are separated by a vector $\vec{r}_{2}$. Note that the magnet with magnetization vector $\vec{m}$ is the main magnet and $\vec{m}$ is perpendicular to $\vec{m}_{2}$. The COM is assumed to be located at the main magnet and thus the magnetic torque and force acting on the microrobot can be expressed by

$$
\left[\begin{array}{l}
\vec{T} \\
\vec{F}
\end{array}\right]=\left[\begin{array}{c}
\operatorname{sk}\left(\vec{m}+\vec{m}_{2}\right) \mathbf{B}+\operatorname{sk}(\vec{r})\left[\begin{array}{c}
\vec{m}_{2}^{\mathrm{T}} \mathbf{B}_{x} \\
\vec{m}_{2}^{\mathrm{T}} \mathbf{B}_{y} \\
\vec{m}_{2}^{\mathrm{T}} \mathbf{B}_{z}
\end{array}\right] \vec{I}^{\mathrm{T}} \mathbf{B}_{x}+\vec{m}_{2}^{\mathrm{T}} \mathbf{B}_{x} \\
\vec{m}^{\mathrm{T}} \mathbf{B}_{y}+\vec{m}_{2}^{\mathrm{T}} \mathbf{B}_{y} \\
\vec{m}^{\mathrm{T}} \mathbf{B}_{z}+\vec{m}_{2}^{\mathrm{T}} \mathbf{B}_{z}
\end{array}\right]
$$

Thus, the two-magnet configuration achieves six-DOF actuation with no downsides from the continuous magnetization case. However, it is noted that eq. (11) could be simplified through the addition of more magnets to the configuration in a symmetrical arrangement. Specifically, the contribution of $\vec{m}_{2}$ to the rigid-body force could be cancelled with the addition 
of one more magnet, and the contribution of $\vec{m}_{2}$ could be restricted completely to torque about the axis of $\vec{m}$ with a total of seven magnets.

\section{B. Decoupled Discrete Magnet Configurations}

The coupling between the magnets can be reduced by using a novel three-magnet configuration as shown in Fig. 2(b). The auxiliary magnets and the main magnet are placed collinearly and the main magnet is located at the center. The auxiliary magnets are magnetized in such a way that they are equal and opposite, i.e. $\vec{m}_{2}$ and $-\vec{m}_{2}$. The magnetization vector of the main magnet, $\vec{m}$, is orthogonal to $\vec{m}_{2}$.

Due to the symmetrical arrangement, the net magnetic moment of the microrobot is aligned with the main magnet $\vec{m}$, and the auxiliary magnets contribute only to the rigid-body torque. Magnetic forces on the auxiliary magnets are equal and opposite, thus cancelling out. The actuation is thus written as

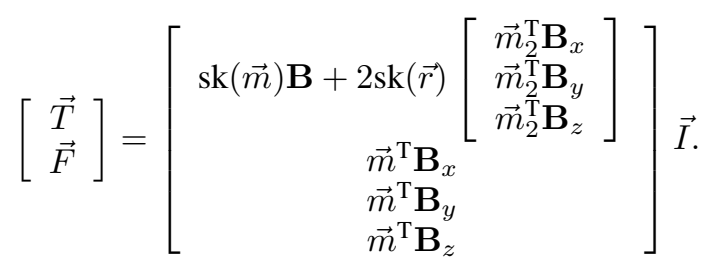

It is noticed that the terms contributing to the force $\vec{F}$ are reduced in this decoupled case. However, the auxiliary magnets are still contributing to torques about all three axes. Mathematically, this means that when $\vec{F}$ is specified,

$$
\operatorname{sk}(\vec{m})\left(\operatorname{sk}(\vec{r})\left[\begin{array}{lll}
\mathbf{B}_{x}^{\mathrm{T}} \vec{m}_{2} & \mathbf{B}_{y}^{\mathrm{T}} \vec{m}_{2} & \mathbf{B}_{z}^{\mathrm{T}} \vec{m}_{2}
\end{array}\right]^{\mathrm{T}} \vec{I}\right) \neq \overrightarrow{0} .
$$

This coupling torque, however, can be eliminated if more auxiliary magnets can be added to the configuration in a symmetrical way. This will mean that the auxiliary magnets are only contributing to the torque about the main magnet magnetization axis $\vec{m}$. This leads to a very intuitive actuation scheme, where the main magnet acts to drive five-DOF as it would in a traditional magnetic microrobot, and the auxiliary magnets only acts to drive the sixth-DOF (torque about the main magnet axis). Note that due to the coupling effects of the magnetic spatial gradients resultant from Gauss's law and Ampere's law, not every configuration with a net magnetization in the direction of $\vec{m}$ can achieve this.

One possible arrangement that can accomplish this full decoupling is the seven-magnet configuration where six discrete magnets are arranged symmetrically along all three axes. There are several seven-magnet configurations that can achieve full decoupling, one of which is shown in Fig. 2(c). Based on this configuration, the net force and torque exerted on the microrobot can be expressed as

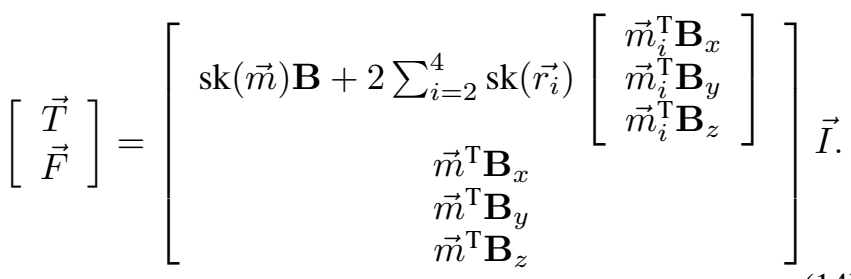

The main difference between eq. (14) and eq. (12) is that the torque generated by the auxiliary magnets can be computed to be parallel to the $\vec{m}$. Mathematically, this means that even when $\vec{F}$ is specified,

$$
\operatorname{sk}(\vec{m})\left(\sum_{i=2}^{4} \operatorname{sk}\left(\vec{r}_{i}\right)\left[\begin{array}{lll}
\mathbf{B}_{x}^{\mathrm{T}} \vec{m}_{i} & \mathbf{B}_{y}^{\mathrm{T}} \vec{m}_{i} & \mathbf{B}_{z}^{\mathrm{T}} \vec{m}_{i}
\end{array}\right]^{\mathrm{T}} \vec{I}\right)=\overrightarrow{0}
$$

This implies that the torque generated by the auxiliary magnets would only contribute to rotational torque about $\vec{m}$ which is the sixth-DOF.

Although the seven-magnet configuration decouples the actuation of the main and auxiliary magnets, the fabrication of such an arrangement would be difficult in practice. Indeed, the decoupled arrangements do not add any actuation capability over the two-magnet configuration, but only serve as interesting cases for study.

\section{CONTROL}

As discussed in section II, to apply a desired six-DOF wrench to the rigid body, the three-DOF orientation of the body must be known, preferably through feedback. If the threeDOF orientation is known, a six-DOF wrench can be applied using eq. (9). However, the requirement for such orientation feedback is burdensome in an experimental implementation. For the previously-established five-DOF control, such a limitation is overcome by commanding the field direction rather than specifying desired torques. The assumption is made that the magnetization direction of the microrobot will quickly orient to the direction of the field. The advantage of this over torque-based control is that the orientation of $\vec{m}$ need not be tracked. As this specifies the vector $\left[\vec{B}^{\mathrm{T}} \vec{F}^{\mathrm{T}}\right]^{\mathrm{T}}$ we call this "BF" mode. The previous section introduced microrobot designs that would allow a microrobot with non-uniform magnetization to be controlled using this control strategy.

To overcome the orientation limitation and incorporate the open-loop strategy of BF mode, one would want to specify the field, rigid-body force, and rigid-body torque. However, these cannot each be specified at the same instant in time. This arises from the fact that the rigid-body torque can be written as a linear combination of individual forces and the applied magnetic field. The actuation matrix would then be overdetermined and not full rank. However, the actuation matrix could be solved for a combination of two of the three desired vectors.

In an open-loop rotation control scheme used in section $\mathrm{V}$ to experimentally confirm six-DOF motion, a torque about the magnetization axis and a desired force is specified. In this case, only the magnetization axis orientation is required. Additional dynamic effects (eg: fluid flow, gravity) could apply a torque on the other two axis of rotation. In addition, the resulting magnetic field will have a contribution to this undesired torque. We designate this "TF" mode as the controller specifies the vector $\left[\stackrel{\vec{T}}{\mathrm{~T}}^{\mathrm{T}} \vec{F}^{\mathrm{T}}\right]^{\mathrm{T}}$.

It is important to reiterate that TF mode can specify torques orthogonal and parallel to the magnetization axis, but without knowledge of the orientation of the affected axis, these could 
produce undesired effects. A second rotation axis could be tracked to give five-DOF control in TF mode, and the third orientation would achieve all six-DOF. In this work when we discuss TF mode, we will be discussing the case of feedback only about the magnetization axis.

We can calculate the resulting field, force, or torque for any set of coil currents $\vec{I}$. We can then calculate the resulting non-zero torque for BF mode and the resulting magnetic field for TF mode. Ideally, these would be zero, except for the case of TF mode, for which we would desire a non-zero field in the direction of the magnetization axis. It is observed that BF mode would contribute non-negligible torque about the magnetization axis, up to $10 \mathrm{~s}$ of $\mathrm{pNm}$ for $200 \mathrm{nN}$ of applied force, which varies depending on the applied field and orientation. This agrees with qualitative observations in previous work. In TF mode, the resulting magnetic field is small compared to typically applied field magnitudes (1-8 mT), however the direction of the field deviated only slightly from the magnetization axis. This indicates TF mode will keep the direction of the magnetization axis. However, in experiments, the field is non-uniform and $\vec{I}$ is solved for the workspace center. The small field magnitude and large gradients generated to produce the torque and force mean that there is a high probability the microrobot will not experience the correct lowmagnitude resultant field.

It is also important to note the remaining combination of desired vectors which could define a control mode, that of a field and a torque, which would be designated "BT mode." This mode would ensure the magnetization axis would be fixed and would specify the torque about it. Under the current control strategy, this would be redundant as the TF mode will generally keep the direction of the magnetization constant. Tests of this mode showed that for typical fields and torques, magnetic forces are an order of magnitude lower than typically desired forces (hundreds of $\mathrm{nN}$ ).

\section{EXPERIMENTS}

\section{A. Fabrication and Actuation}

Microrobots containing discrete permanent magnets are fabricated in several pieces and fixed together using an adhesive. Individual magnet elements are fabricated to be magnetically hard, retaining their internal magnetization in the absence of an externally applied magnetic field. Microrobots are fabricated in a batch process using soft photolithography and molding techniques in a manner similar to techniques for micro-scale robots and parts [2, 8]. The shape is chosen as rectilinear solid, but could be chosen arbitrarily to suit a particular application. Microrobot magnetic elements are composed of neodymium-iron-boron $(\mathrm{NdFeB})$ particles in a polyurethane matrix. Due to the high magnetic coercivity of magnetized $\mathrm{NdFeB}$ (i.e. fields over $600 \mathrm{mT}$ are required to demagnetize $\mathrm{NdFeB}$ ), these microrobots are not subject to demagnetization from the relatively weak fields applied in this work. The molding process for the magnetic elements is prone to variations in microrobot geometry (up to about $10 \%$ from nominal), but the control method is not sensitive to these small geometric changes. To achieve decoupled actuation, each magnetic element must have the same magnetic moment strength.
This is ensured by magnetizing each element individually in an alternating gradient force magnetometer (AGFM, Micromag $2900)$ to a strength of $1.64 \pm 0.01 \mu \mathrm{Am}^{2}$.

The discrete magnetic elements are mounted to a molded polyurethane base or an acetal base which is laser-cut from a flat sheet. The nature of the material is not important from a magnetic actuation standpoint, but does provide a base upon which to precisely align the magnetic elements, and offers a shape for computer vision detection. The shape chosen for experimental demonstrations is shown in Fig. 3, and consists of a circle with a flat edge for ease of image processing. The relative orientation of each magnet element in the microrobot is critical to achieve accurate control based on eq. (12), and so the magnets are placed one-at-a-time with a magnetic alignment jig, as shown in Fig. 3(a) for the example microrobot with reduced coupling shown in Fig. 2(b). This design consists of three magnetic elements which must be aligned perpendicular and anti-parallel to each other. In Fig. $3(\mathrm{a}, \mathrm{i})$, the first module is placed pointing to the right. The jig magnets provide an aligning field to ensure that the element is aligned with minimal error. The module is held in place with UV-curable adhesive (Loctite 3761) so that it can be cured with a UV light source after alignment is ensured. In Fig. 3(a,ii), the jig magnets are reversed and the second magnet is placed and fixed. In Fig. 3(a,iii), an out-of-plane jig magnet is used to place the final magnetic element pointing in the upward direction. The magnets are aligned by eye such that all three magnets are collinear with each other and the flat of the base. Once all magnetic elements are placed, the microrobot is removed from the jig and can be placed in a liquid environment for actuation. A photograph of the aligning jig is shown in Fig. 3(b) and the completed microrobot is shown in Fig. 3(c).

The magnetic microrobots are actuated by the eight independent air or iron-core electromagnetic coils shown in Fig. 4, which are aligned pointing to a common workspace center point with an approximate opening size of $12 \mathrm{~cm}$. The currents in the electromagnetic coils are controlled using a PC with a data acquisition system at a control bandwidth of $20 \mathrm{kHz}$, using linear electronic amplifiers (SyRen 25, Dimension Engineering Inc.) and Hall-effect current sensors (ACS714, Allegro Microsystems Inc.). Imaging of the microrobots and workspace is accomplished by two CCD cameras (Foculus F0134SB) connected to variable magnification microscope lenses, providing up to a $26 \mathrm{~mm} \times 20 \mathrm{~mm}$ field of view from the top and side perspectives. Magnetic flux densities and gradients of up to $8.3 \mathrm{mT}$ and $0.34 \mathrm{~T} / \mathrm{m}$, respectively, can be generated in the workspace, with a $3.3 \times$ increase when iron cores are inserted into the coils. For a $20 \mathrm{~mm} \times 20 \mathrm{~mm} \times 20 \mathrm{~mm}$ workspace in the center of the coil system, the field and field gradient are shown to be uniform within $6.0 \%$ of the nominal values as measured with a Hall effect sensor (Lakeshore Gaussmeter 410).

\section{B. Orientation Detection}

The orientation of the microrobot about the magnetization axis is accomplished using visual feedback with OpenCV software. Hough transforms are used to identify the center of the circular shape and the endpoints of the flat edge of the microrobot body. To ensure precise and accurate object detection, the workspace is backlit to create a high-contrast 
(a)

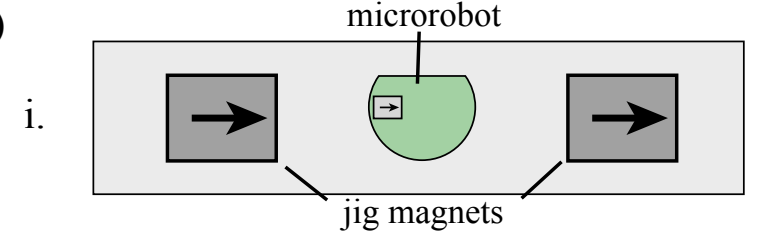

ii.

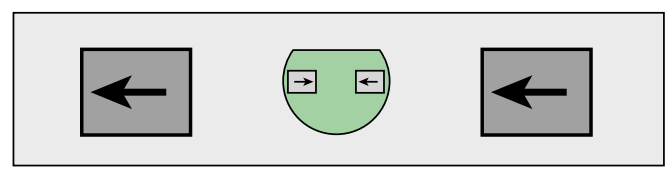

iii.

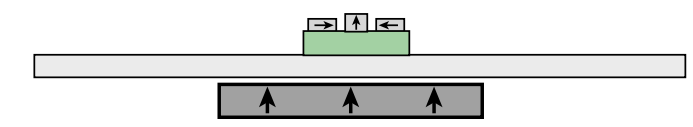

(b)

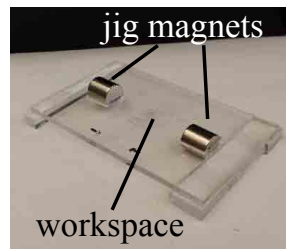

(c)

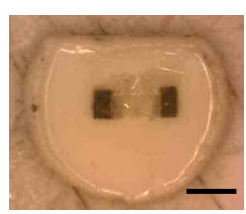

Fig. 3. (a) Module assembly. i) First magnet is affixed to the microrobot body and aligned. ii) Jig magnets are flipped and the second magnet is affixed to the body. iii) The jig magnets are removed, a magnet is placed underneath the jig and the center magnet is affixed to the body. (b) Assembly jig with alignment magnets. (c) Assembled module with two magnets of opposing magnetization. Note that the arrows represent the magnets' magnetization vector. Scale bar is $1 \mathrm{~mm}$.

silhouette of the microrobot for the top-camera view. The detection algorithm returns the center position of the microrobot and its orientation once per video frame. For the experiments shown in this work, we assume that the microrobot is oriented so that the top or side camera is directly viewing one of the flat faces of the microrobot. This constraint could be relaxed for general rotations if both cameras are used simultaneously for orientation detection.

\section{Experimental Results}

1) Water Surface Actuation Results: By eq. (12), a commanded torque about the magnetization axis should not result in any linear motion. However, fabrication errors will cause a commanded linear or rotational motion to induce an undesired motion due to magnet misalignments, we call this "actuation coupling". As the magnetization axis was aligned with the $z$-direction for orientation detection, the actuation coupling between the torque about the $z$-axis and the linear motions in the $x$ - and $y$-directions was investigated. The microrobot was actuated using the TF control method on the water surface. This was done to restrict rotation about the axes normally controlled by open loop five-DOF control which can yield disturbances in TF mode due to the resulting magnetic field as discussed in section IV. The water surface also restricts $z$-axis motion, and reduces surface interactions which affect motion of the microrobot.

To minimize surface energy, a meniscus forms on the (a)

(b)

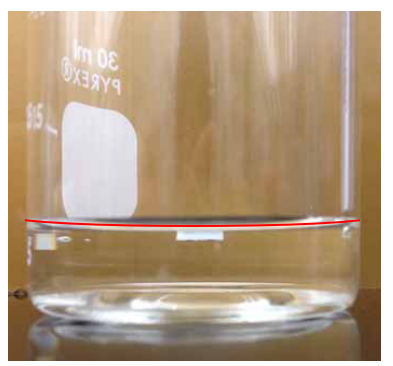
top camera

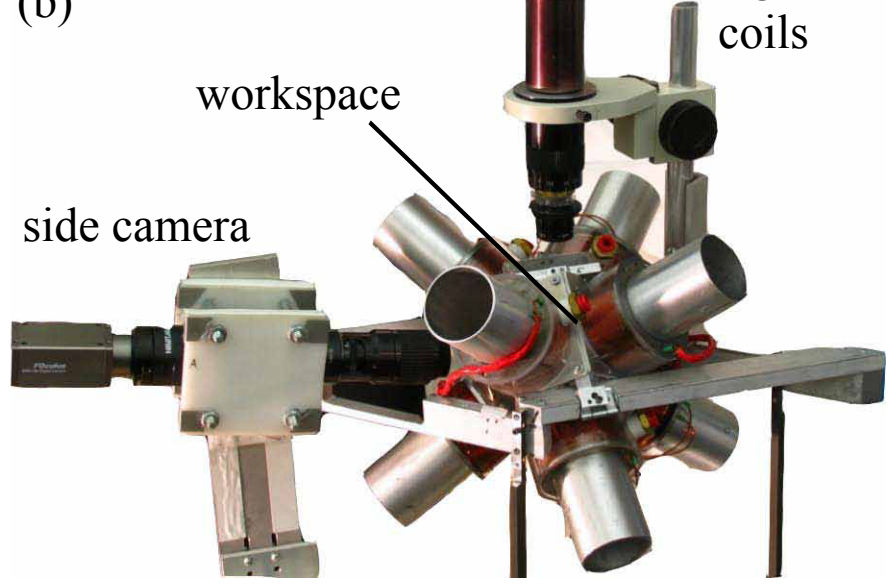

Fig. 4. (a) Shape of water meniscus. The meniscus shape is predicted by eq. (16). (b) Photograph of the electromagnetic coil setup. All system components are shown with the coils in the operational position. Two coils hinge open to allow for access to the workspace. The workspace is a $20 \mathrm{~mm} \times 20 \mathrm{~mm} \times 20 \mathrm{~mm}$ cube in the center of the electromagnetic coils.

water-air interface and satisfies the linearized Laplace equation, which is solved by

$$
h(x, y)=x_{c} \cot \left(\theta_{c}\right) \operatorname{csch}\left(\frac{R}{x_{c}}\right) \cosh \left(\frac{\sqrt{x^{2}+y^{2}}}{x_{c}}\right),
$$

where $x_{c}$ is the characteristic capillary length, $\theta_{c}$ is the contact angle of the container (glass), and $R$ is the radius of the cylindrical container [7].

The meniscus opposes displacement of the microrobot on the water surface. When the microrobot is at a steady state displacement, the forces of the meniscus on the microrobot are

$$
\begin{aligned}
& F_{x}(x, y)=m_{r} g \frac{\partial h(x, y)}{\partial x} \\
& F_{y}(x, y)=m_{r} g \frac{\partial h(x, y)}{\partial y},
\end{aligned}
$$

where $m_{r}$ is the effective mass of the microrobot.

When rotating, the microrobot will experience drag due to being on the water surface and follows the drag form equation,

$$
\vec{T}_{d}=C_{d} \vec{\omega}^{2}
$$

where $\omega$ is the rotational velocity of the microrobot. The drag coefficient, $C_{d}$, is found to be $4.2 \times 10^{-10} \pm 5.4 \times 10^{-22} \mathrm{Nms}^{2}$ by a first order least squares fit of the observed $\omega^{2}$ for a given torque. Variations in 

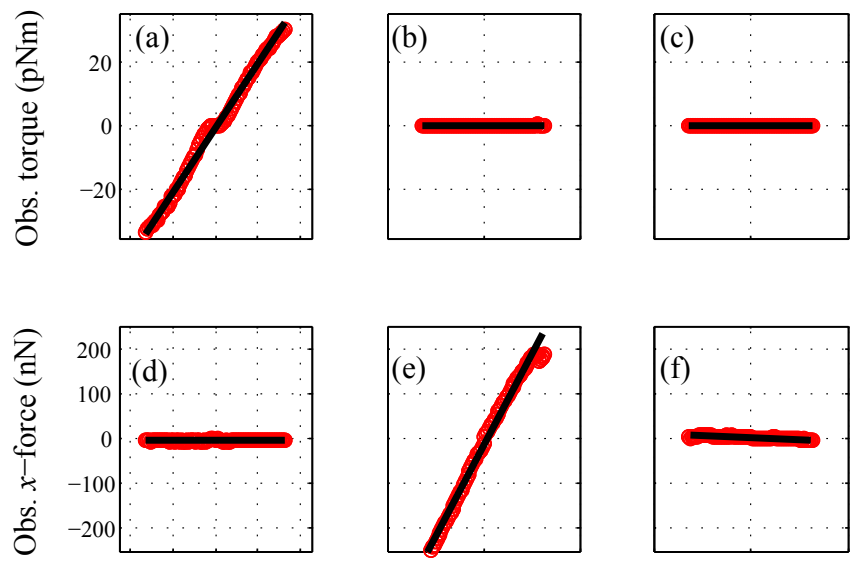

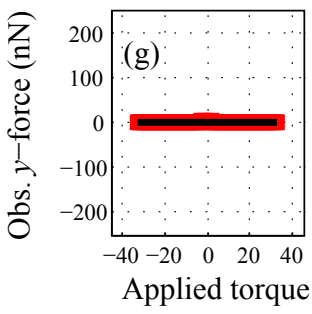

$(\mathrm{pNm})$

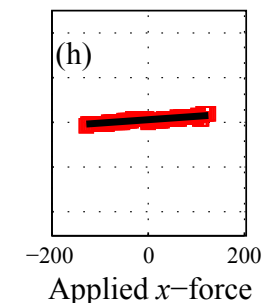

$(\mathrm{nN})$

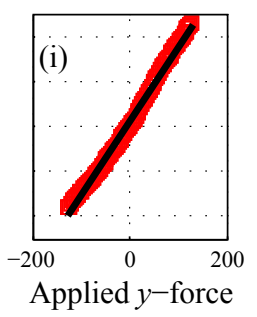

$(\mathrm{nN})$
Fig. 5. Actuation coupling of the forces in the $x$ - and $y$-directions and torque about the $z$-axis for a microrobot operating on the water surface(red) and linear fits corresponding to eqs. $(17,18)$ (black). Each plot corresponds to an element in eq. (19). The rows correspond to the torque (a-c), $x$-force (d-f), and $y$-force (g-i) response to an independent desired torque (left column), $x$-force (center column), and $y$-force (right column). In this example $\vec{m}=0.64, \vec{m}_{2}=1.64$ $\pm 0.01 \mu \mathrm{Am}^{2}$. The error bars of each measurement cannot be seen due to their size, $2.76 \pm 1.58 \mathrm{nN}$ for displacement actuation and $0.03 \pm 0.11 \mathrm{pNm}$ for rotation actuation.

the drag coefficient will affect the magnitude of the observed torque for a given observed rotation rate, but will not affect the observed decoupling of actuation between the torque and the translation forces.

In the experiment, the controller swept through steps of forces or torques. At each force or torque, $5 \mathrm{~s}$ was allowed for steady-state position or rotation rate to be achieved, and the state was recorded for 5 seconds for forces and 25 seconds for torques, each at a rate of 30 samples/second. Between each experiment, $60 \mathrm{~s}$ was allowed for the coils to cool. Figure 5 shows the results of independently applied torque about the $z$-axis, and force in the $x$ - and $y$-directions and the observed resulting forces and torque. An ideal microrobot would produce plots with slopes of 1 in the diagonal plots and slopes of 0 on the off-diagonal plots. As the forces and torques on the water surface are difficult to model accurately, experimental error is expected. The model is very sensitive to environment parameters, especially the container-water contact angle, microrobot-water contact angle, and microrobot weight. However, errors in these parameters would scale the observed forces and torques proportionally across an experiment. In the experimental analysis, we are primarily interested in the ratio between desired and undesired actuations, so these proportional scalings will not affect the results.
We study the ratio of these actuations by looking at the magnitude of each observed motion when one actuating force or torque is desired. This is represented by the actuation coupling matrix,

$\left[\begin{array}{lll}\frac{\partial T_{\theta}}{\partial T_{\theta}} & \frac{\partial T_{\theta}}{\partial F_{x}} & \frac{\partial T_{\theta}}{\partial F_{y}} \\ \frac{\partial F_{x}}{\partial T_{\theta}} & \frac{\partial F_{x}}{\partial F_{x}} & \frac{\partial F_{x}}{\partial F_{y}} \\ \frac{\partial F_{y}}{\partial T_{\theta}} & \frac{\partial F_{y}}{\partial F_{x}} & \frac{\partial F_{y}}{\partial F_{y}}\end{array}\right]$.

The diagonal terms of the actuation coupling matrix represent the desired motions, while the off-diagonal terms represent undesired motions due to the desired motion. When the microrobot magnetization $\vec{M}(\vec{r})$ is known and eq. (9) is solvable, then the actuation coupling matrix should be the identity matrix and the motions completely decoupled. The scale of the offdiagonal terms give the actuation coupling of the system. Thus, we can look at the relative magnitude of the diagonal and off-diagonal terms to determine the precision of actuation. To allow for comparison of forces with torques in the actuation coupling matrix, the elements of eq. (19) are scaled to compare a force to a force. The terms $\frac{\partial T_{\theta}}{\partial F_{x}}$ and $\frac{\partial T_{\theta}}{\partial F_{y}}$ are scaled by dividing the torques by the length of the moment arm of the microrobot, $2 \mathrm{~mm}$. The observed forces that are due to forces, $\frac{\partial F_{x}}{\partial T_{\theta}}$ and $\frac{\partial F_{y}}{\partial T_{\theta}}$, are multiplied by the length of the moment arm. To further aid comparison, each row of the matrix is individually scaled such that each diagonal term is unity, eliminating error from the rotational drag and meniscus models. The slopes of the fitted lines in Fig. 5 correspond to the actuation coupling matrix, which is found to be

$$
\left[\begin{array}{lcr}
1 & 1.3 \times 10^{-4} & 2.7 \times 10^{-5} \\
1.9 \times 10^{-11} & 1 & -1.7 \times 10^{-2} \\
-1.5 \times 10^{-11} & 4.9 \times 10^{-2} & 1
\end{array}\right] \text {. }
$$

The worst actuation coupling factors are the forces in $x$ and $y$ that correspond to the desired $y$ - and $x$-forces, respectively. These errors can be due to the sensitivity in the camera rotation with respect to the workspace. Despite this, the worst-case actuation coupling is that of the $x$-displacement response when experiencing a $y$-force, with a response 20 times less than that of the $y$-displacement response.

2) Six-DOF Actuation: A second, near-neutrally buoyant microrobot was used to qualitatively demonstrate unconstrained six-DOF control in a fluid. The 3-magnet microrobot was fabricated as described in section V-A, with the exception that the polymer body was fabricated to have a density less than that of water by inclusion of hollow glass microbeads (3M K1). Additional weights were manually added to the underside of the microrobot (the side with the aligned magnets) until the overall density approached that of high viscosity 50 cSt silicone oil. The high viscosity oil was used to slow the motions for easier observation in this demonstration. Independent actuation of each DOF was then applied one-at-a-time to the microrobot and recorded. Here, visual feedback was only required for torque about the $z$-axis.

Snapshots of the motions can be seen in Fig. 6. The left hand side shows the side camera views for rotation about the $x$ - and $y$-axes and $z$-axis translation. The right hand side shows the top camera view for $x$ - and $y$-axes translation and rotation about the $z$-axis. An outline of the neutral starting position is 

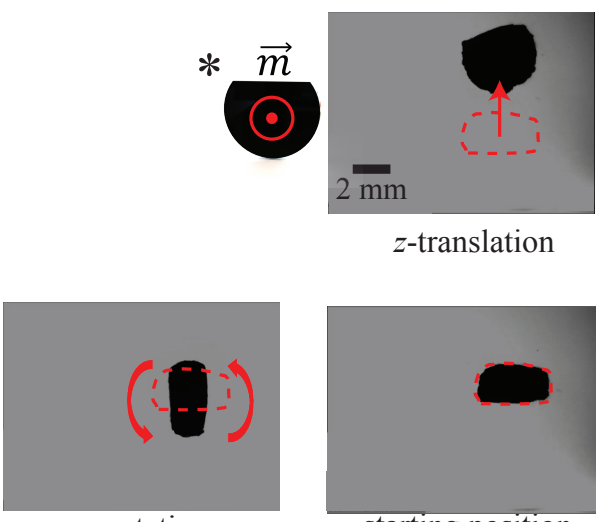

$x$-rotation

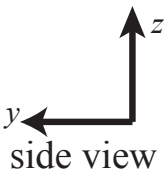

side view

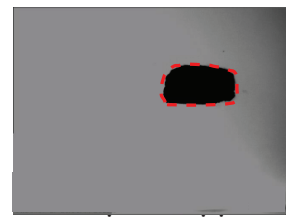

starting position

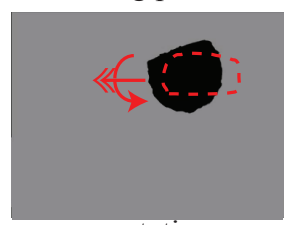

$y$-rotation

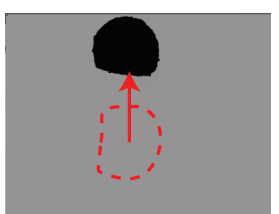

$y$-translation

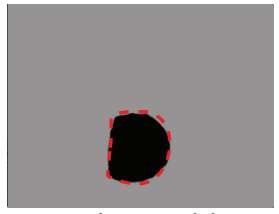

starting position

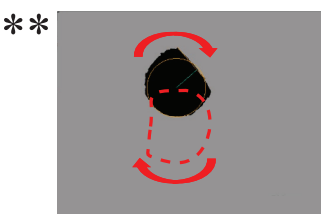

$z$-rotation
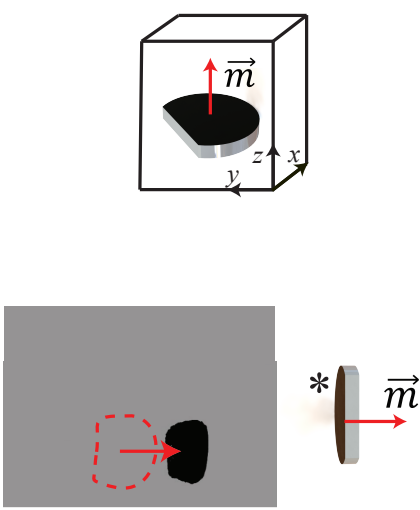

$x$-translation

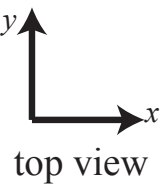

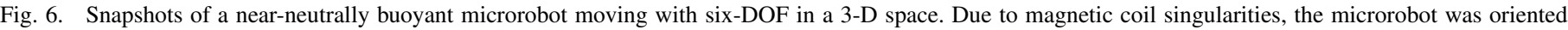

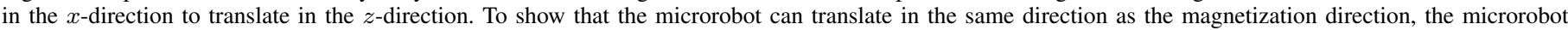

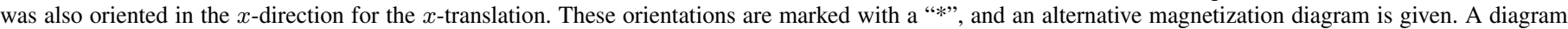

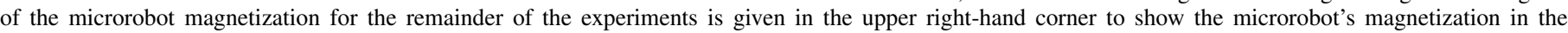

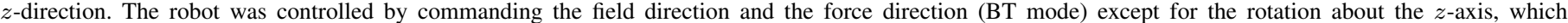

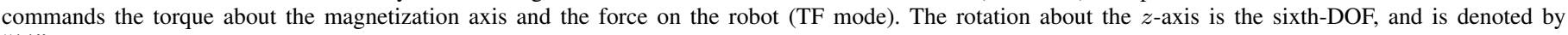
“***.

given for reference. The $y$ - and $z$-translations exhibit minor rotation about the magnetization axis, which is expected as there is no control about that axis. The various rotations exhibit minor $x$ - and $y$-motions. All motions except for the controlled $z$-translation gradually move in the negative $z$-direction due to gravity. Due to the higher current requirements of applying fields and forces in the same direction [12], the microrobot is oriented in the $x$-direction for the $x$ - and $z$-translations, and the $z$-direction for the $y$-translation. The only feedback used is to determine the direction of $\vec{m}_{2}$ for the rotation about the magnetization axis. This is used as input for the TF controller, which is used for rotation about the $z$-axis. The remainder of the motions are controlled via a teleoperated open loop controller and utilize the $\mathrm{BF}$ controller. As the polymer body is fabricated to have a density less than the oil and the magnetic masses, which have a density greater than that of oil, are on one side of the body, buoyant forces allow for the microrobot to "self-right". This is beneficial for experiments as the microrobot would naturally align itself such that the primary magnet would point towards the top camera for angle detection for the rotation about the $z$-axis. However, the magnetic torque has to overcome this force when aligning to the magnetic field in a different direction. The translational motions behaved as expected, with minimal undesired motion. However, as expected, there does exist some rotation about the magnetization axis, which is uncontrolled in BF mode, and is most clearly seen in the $z$-translation. The rotation about the $z$-axis was successful, however only one rotation in either the clockwise and counterclockwise direction could be accomplished before the microrobot would reach the bottom of the work environment. This is due to the slow rotation about the magnetization axis.

\section{CONCLUSIONS}

We have introduced a theoretical framework and the design requirements to achieve six-DOF actuation of a magnetic microrobot. A general guideline to achieve this actuation is to have a non-uniform magnetization profile with components which are non-parallel to the net magnetization vector of the microrobot. As previous magnetic microrobot actuation schemes cannot achieve torque about the microrobot magnetization axis, we have shown full six-DOF magnetic actuation for the first time. We demonstrated the accuracy of the theoretical model through reduced-DOF water-surface experiments, demonstrating a high level of decoupling between actuation DOF. We also demonstrated full six-DOF actuation for a microrobot in liquid in a proof-of-concept demonstration. Controlled translation and orientation control of a mobile microrobot with six-DOF will require feedback of microrobot orientation and implementation of a controller which can suppress the small levels of actuation error arising from imprecise microrobot fabrication and magnetization. Future work will detail further microrobot feedback methods using vision or other methods for six-DOF control. This actuation strategy will be applied to microrobot tasks which benefit from full position and orientation control such as 3-D manipulation and assembly of micro-objects [20].

\section{REFERENCES}

[1] E. Diller and M. Sitti. Micro-scale mobile robotics. Foundations and Trends in Robotics, 2(3):143-259, 2013. 
[2] E. Diller, C. Pawashe, S. Floyd, and M. Sitti. Assembly and disassembly of magnetic mobile microrobots towards deterministic 2-D reconfigurable microsystems. International Journal of Robotics Research, 30 (14):1667-1680, 2011.

[3] E. Diller, S. Miyashita, and M. Sitti. Remotely addressable magnetic composite micropumps. RSC Advances, 2 (9):3850-3856, 2012.

[4] E. Diller, J. Giltinan, and M. Sitti. Independent control of multiple magnetic microrobots in three dimensions. International Journal of Robotics Research, 32(5):614631, 2013.

[5] C. Elbuken, M. Khamesee, and M. Yavuz. Design and implementation of a micromanipulation system using a magnetically levitated MEMS robot. IEEE/ASME Transactions on Mechatronics, 14(4):434-445, 2009.

[6] D. R. Frutiger, K. Vollmers, B. E. Kratochvil, and B. J. Nelson. Small, fast, and under control: wireless resonant magnetic micro-agents. International Journal of Robotics Research, 29(5):613-636, 2009.

[7] K. Hosokawa, I. Shimoyama, and H. Miura. Twodimensional micro-self-assembly using the surface tension of water. Sensors and Actuators A: Physical, 57(2): $117-125,1996$.

[8] M. Imbaby, K. Jiang, and I. Chang. Net shape fabrication of stainless-steel micro machine components from metallic powder. Journal of Micromechanics and Microengineering, 18(11):115018, 2008.

[9] I. S. Khalil, V. Magdanz, S. Sanchez, O. G. Schmidt, and S. Misra. The control of self-propelled microjets inside a microchannel with time-varying flow rates. IEEE Transactions on Robotics, 2013.

[10] M. Khamesee, N. Kato, Y. Nomura, and T. Nakamura. Design and control of a microrobotic system using magnetic levitation. IEEE/ASME Transactions on Mechatronics, 7(1):1-14, 2002.

[11] S. Kim, F. Qiu, S. Kim, A. Ghanbari, C. Moon, L. Zhang, B. J. Nelson, and H. Choi. Fabrication and characterization of magnetic microrobots for three-dimensional cell culture and targeted transportation. Advanced Materials, 25(41):5863-5868, 2013.

[12] M. Kummer, J. Abbott, B. Kratochvil, R. Borer, A. Sengul, and B. Nelson. OctoMag: An electromagnetic system for 5-DOF wireless micromanipulation. IEEE Transactions on Robotics, 26(6):1006-1017, 2010.

[13] A. W. Mahoney, D. L. Cowan, K. M. Miller, and J. J. Abbott. Control of untethered magnetically actuated tools using a rotating permanent magnet in any position. Robotics and Automation (ICRA), 2012 IEEE International Conference on, pages 3375-3380, 2012.

[14] S. Martel, J.-B. Mathieu, O. Felfoul, A. Chanu, E. Aboussouan, S. Tamaz, P. Pouponneau, L. Yahia, G. Beaudoin, G. Soulez, and M. Mankiewicz. Automatic navigation of an untethered device in the artery of a living animal using a conventional clinical magnetic resonance imaging system. Applied Physics Letters, 90(11):114105, 2007.

[15] S. Martel, M. Mohammadi, O. Felfoul, Z. Lu, and P. Pouponneau. Flagellated magnetotactic bacteria as controlled MRI-trackable propulsion and steering systems for medical nanorobots operating in the human microvasculature. International Journal of Robotics Research, 28(571-582), 2009.

[16] D. Meeker, E. Maslen, R. Ritter, and F. Creighton. Optimal realization of arbitrary forces in a magnetic stereotaxis system. IEEE Transactions on Magnetics, 32 (2):320-328, 1996.

[17] M. Miyasaka and P. Berkelman. Magnetic levitation with unlimited omnidirectional rotation range. Mechatronics, 24(3):252-264, 2014.

[18] M. S. Sakar, E. B. Steager, D. H. Kim, A. A. Julius, M. Kim, V. Kumar, and G. J. Pappas. Modeling, control and experimental characterization of microbiorobots. International Journal of Robotics Research, 30(6):647-658, 2011.

[19] M. Sitti. Voyage of the microrobots. Nature, 458:11211122, 2009.

[20] S. Tasoglu, E. Diller, S. Guven, M. Sitti, and U. Demirci. Untethered micro-robotic coding of three-dimensional material composition. Nature Communications, 5, 2014. doi: $10.1038 /$ ncomms4124.

[21] S. Tottori, L. Zhang, F. Qiu, K. K. Krawczyk, A. FrancoObregón, and B. J. Nelson. Magnetic helical micromachines: fabrication, controlled swimming, and cargo transport. Advanced Materials, 24(6):811-816, 2012.

[22] P. Vartholomeos, M. R. Akhavan-sharif, and P. E. Dupont. Motion planning for multiple millimeter-scale magnetic capsules in a fluid environment. In IEEE Int. Conf. Robotics and Automation, pages 1927-1932, 2012.

[23] P. Vartholomeos, C. Bergeles, L. Qin, and P. E. Dupont. An MRI-powered and controlled actuator technology for tetherless robotic interventions. International Journal of Robotics Research, 32(13):1536-1552, 2013.

[24] Z. Ye, E. Diller, and M. Sitti. Micro-manipulation using rotational fluid flows induced by remote magnetic micro-manipulators. Journal of Applied Physics, 112(6): 064912, 2012. 